\title{
Exploring Electric Driving Pleasure - The BMW EV Pilot Projects
}

\author{
Jens Ramsbrock ${ }^{1}$, Roman Vilimek ${ }^{2}$, and Julian Weber ${ }^{1}$ \\ ${ }^{1}$ BMW Group, Innovation Projects E-Mobility, Munich, Germany \\ ${ }^{2}$ BMW Group, Concept Quality, Munich, Germany \\ \{jens.ramsbrock, roman.vilimek, julian.weber\} abmw. de
}

\begin{abstract}
An electric vehicle (EV) is more than just a car with an electric engine. It implies a major shift in everyday experience. Charging the vehicle at home, thinking about where this energy comes from, dealing with limited range or driving a silent vehicle without engine noise are only some aspects of a completely new ecosystem for an electric vehicle owner. Of course, EVs will only succeed in the mass market if they meet customers' expectations. With the decision to step into this unknown terrain, the BMW Group gathered data in field trials with pilot customers of the MINI E and BMW ActiveE. The field trials discovered that everyday driving does not differ significantly from conventional vehicles in the same segment. About $90 \%$ of intended trips can be realized, showing the gap that needs to be closed is manageable. In order to close it, BMW will offer innovative mobility services and charging solutions.
\end{abstract}

Keywords: Electric Vehicle, MINI E, BMW ActiveE, field trial, user study.

\section{The Road towards Series-Produced Electric Vehicles}

Global developments like climate change, decreasing availability of natural resources and increasing urbanization call for new solutions in many facets of everyday life. This demand implies also the need for changes in mobility patterns. Within the BMW Group, the sub-brand BMW i was created to respond to this situation. The goal is to design purpose-built electric vehicles and mobility services to support sustainable individual mobility. For the BMW i3, BMW Group's first series-produced EV, the life cycle global warming potential $\left(\mathrm{CO}_{2 \mathrm{e}}\right)$ is at least a third lower than for a state-ofthe-art highly efficient combustion-engine vehicle in the same segment ${ }^{1}$. If the vehicle is charged with renewable energy, this can be increased up to well over 50 per cent. Recycled materials in the vehicle interior and exterior play a major role, as well as weight savings by extensively using innovative materials like CFRP (carbon fibrereinforced plastic).

The focus on sustainability is not restricted to the operation of the vehicle itself but widened over the complete value chain. The BMW i3 will lead to a massive increase

1 Estimated for an i3 concept car under the assumption of the European electricity mix (EU25). 
in the already high standards of BMW's sustainable production system. Recycling of materials used during the production process will be carried out wherever possible. These high standards are also laid upon the supply chain.

As the mobility needs of people will change when switching to electric vehicles with limited driving range, mobility services will also be offered as a part of the BMW i solution portfolio. On-demand vehicle solutions like DriveNow, a joint venture between the BMW Group and Sixt AG, and innovative functions within the vehicle itself and/or as Apps on smartphones target the requirements of urban personal mobility.

These offers lay a solid foundation to establish electric vehicles as a means of sustainable individual mobility. Of course customers will only accept them if they meet their needs and expectations. BMW launched a project in 2007 to perform thinking outside of the box and explore sustainable mobility solutions, called the project $i$. Analyzing EV customers with a conventional market research approach was not possible in the early phases, which was project $i$ 's main intention to make this feasible. Hardly any EV customers were on the road and the interdependencies between the infrastructure and the car itself have not even been touched by large-scale research.

Therefore field trials with conversion electric vehicles were set up in cooperation with expert partners from universities and research institutions for scientific monitoring and with partners from the infrastructure sector as well as the energy sector. The trials were conducted in several countries in close collaboration and exchange with the local public authorities in order to assess long-term developments in policy towards e-mobility. These field trials are unparalleled worldwide in their scope. Starting with the first step in 2009 in the United States and in Germany, private and corporate pilot customers rented EVs from a fleet of over 600 MINI E cars. The MINI E is a conversion of the MINI Cooper developed to assess the general feasibility and acceptance of an EV as a megacity vehicle. Between 2009 and 2012, data from more than 16 million kilometers (10 million miles) driven by customers in the United States, Germany, the United Kingdom, France, Japan and China were gathered. More than 15,000 people applied to rent a MINI E, 430 private households took part in extensive mostly face-to-face interviews and 14 fleet user companies actively participated in the study. At the end of 2011 the second step of the learning projects for BMW i began. A fleet of over 1,000 BMW ActiveE vehicles, conversions of the BMW 1 Series Coupe, set for launch in several countries. This part of the field trials focuses more directly on technological innovations for EVs. For instance, the study will deliver customer feedback on using remote functions via smartphone apps, pioneering driver assistance systems and switching between comfort-oriented and efficiency-oriented driving with the so-called ECO PRO mode. In contrast to the MINI E field trial that analyzed fundamental aspects of everyday life with electric vehicles, the BMW ActiveE studies have their goals analog to usability tests: Identify specific optimization potential of certain (pre-production) functions. In both field trials an accompanying social media analysis was carried out. While the comments of pilot customers in blogs, newsgroups or social networks were used mainly to inform our research partners about issues from a direct customer's perspective in order to guide the design of quantitative methods, regular social media reports were additionally used during the BMW ActiveE field 
trials to assess the reaction of customers on newly introduced features. The transfer from sometimes even singular reactions in social media to a decision process in the development of vehicles is detailed in [1].

In the following, firstly the key results of the MINI E field trials are summarized first in a very condensed form. Secondly, already available data from the ActiveE study in Germany and the US are discussed. As the data reported here stems from field trials which are stretched massively over time (2009-2013) and which witnessed considerable changes during this time, the results should be regarded as tendencies. Only descriptive statistics are provided. If not otherwise state, numerical values (\% agreement) refer to answers on a Likert scale from 1 (do not agree at all) to 6 (fully agree) and are dichotomized to top three ("agree) and bottom three ("disagree") judgments.

\section{The MINI E Study}

The MINI E is a two-seat development of the MINI hatch featuring a $204 \mathrm{hp} \mathrm{(150}$ $\mathrm{kW}$ ) electric motor that develops a torque of $220 \mathrm{Nm}$. The air-cooled $35 \mathrm{kWh}$ Litihium-Ion battery consumes most of the luggage department as well as the space of the back seats. The range in real terms is up to $130-160 \mathrm{~km}$ (80-100 miles). Charging with 32 ampere takes about 4 hours or 10 hours with 12 ampere respectively.

In terms of user interface, the dashboard was adapted rather minimally to display EV specific functions as shown in Figure 1. The battery level indicator is mounted behind the steering wheel replacing the traditional rev counter. It shows in percentage figures how much charge is still available in the battery. The small display below the indicator allows to switch between a small set of additional items like battery temperature, consumption or charging voltage. The central gauge in the middle of the dashboard displays the current velocity. It also includes an LED display, the converted fuel gauge, indicating power consumption or regeneration. The display and control options of the MINI E are sufficient for the basic interaction with EV functions. However, the customers strongly demanded more detailed information on consumption and EV specifics as well as more options to control advanced EV functions like being able to set charging times or to remotely monitor the charging progress. These requests were fed into the development process and resulting solutions in the BMW ActiveE are again subject to customer test in field trials.

The MINI E field trial served to address key questions on everyday usage patterns, expectations, reactions to special and typical electric vehicle characteristics and additional aspects like the perception of the importance of ecological added value of EV driving to customers. Several universities and research institutions were involved in the field trial. Research partners were the Chemnitz University of Technology (Germany), the University of California at Davis (USA), Oxford Brookes University (UK), the Institute of Science and Technology for Transport, Development and Networks (IFSTTAR, France), the Waseda University and the marketing research company IID (Japan) and the Chinese Automotive Technology and Research Center as well as the market research company INS (China). The study was designed to 
maximize the participation on customer's everyday life with the EV. Travel and charging diaries were administered repetitively as well as a several interviews during the usage period [2-3].

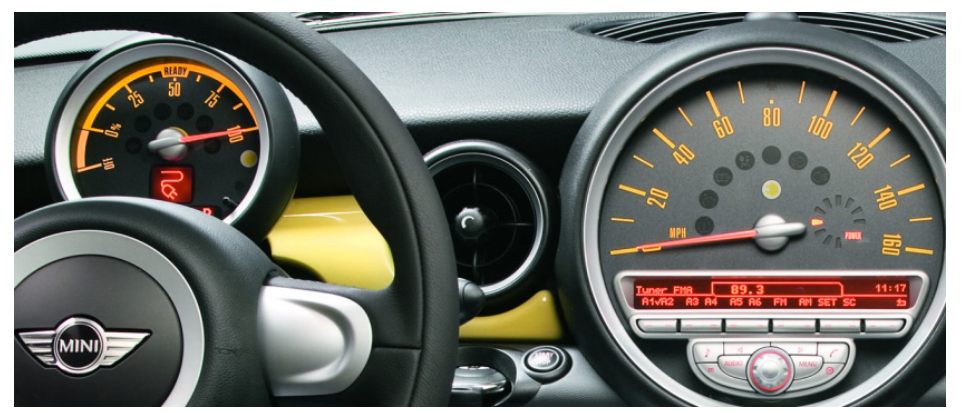

Fig. 1. MINI E dashboard (Fotocredit: BMW Group): Battery level indicator (left) and central gauge (right)

Potential customers interested in driving the MINI E applied via an online application tool. Besides providing socio-demographic information and giving feedback on their motives and interests in electric driving, applicants needed to fulfill different selection criteria (e.g., be willing to take part in interviews, drive the vehicle on a regular basis, be willing to pay a monthly leasing fee). Background information on the large number of applicants, about 500-3500 per country for each 6-12 months usage period, allowed to form a study sample that was representative for the early adopter generation of EV customers. Details on rationale and procedure are described in [3].

The most important finding from the MINI E field trial was that electric vehicles are suitable for everyday life for a large customer segment. Onboard data logger information was read out from the MINI E cars and compared to conventional vehicles within the same segment (BMW 116i and MINI Cooper). Control group customers with combustion engine vehicles used their cars on average for $43.4 \mathrm{~km}$ (27.0 miles, MINI Cooper) and $42.0 \mathrm{~km}$ (26.1 miles, BMW 116i) per day. The average daily distances for MINI E vehicles range from $38.6 \mathrm{~km}$ (24.0 miles) in Germany over 44.2 $\mathrm{km}$ (27.5 miles) in France to $47.8 \mathrm{~km}$ (29.7 miles) in the UK to $49.0 \mathrm{~km}$ (30.4 miles) in the US and in China.

Customers were able to satisfy about $80 \%$ of their daily mobility needs with the MINI E. Based on the MINI E drivers' subjective estimation and travel diary data, they would have been able to use the vehicle for approximately $90 \%$ of the intended trips if the MINI E had more than two seats and standard storage space. Due to the limitations of the conversion vehicle concept, the MINI E had an extremely small luggage compartment. However, it should be noted that the range of the MINI E was not always available. As air cooling was the only thermal management of the batteries, the MINI E was not able to deliver the full range during cold temperatures in winter. This was regarded as major drawback by customers.

The MINI E study also showed that customers get used to charging procedure and range quickly. After less than one month of usage, customers charge only once every 
two or three days. If customers used a wallbox that allows to charge in less than 4 hours, average charging events per week stabilized at 1.9 (Germany) to 2.9 (UK). Only if charging takes longer (about 9-10 hours with a standard wall socket), customers needed to charge almost daily as data from the France field trial shows.

Regenerative braking is an advanced function for electric vehicles. It refers to using the electric motor as a generator when decelerating by lifting the foot from the accelerator pedal. The deceleration is quite strong with $-2.25 \mathrm{~m} / \mathrm{s}^{2}$ in the MINI E and allows driving the car in most situations with one pedal only after some driving practice is gained. From an efficiency point of view, regenerative braking can be a powerful tool to save energy. To assess customer acceptance, a long-term evaluation was implemented. The customer feedback was overwhelming. After at least three months of usage, between 92\% (China, Japan) and almost 100\% (Germany, UK, US, France) customers unanimously said that they would not want to miss this feature in an EV.

In short, the international MINI E trials demonstrated that an electric vehicle with slightly larger and more stable range as well as more space for passengers and cargo will meet the mobility needs for urban use to a very large extent. A more comprehensive overview over the MINI results is provided in [4].

\section{The BMW ActiveE Study}

Like the MINI E, the BMW ActiveE is a conversion vehicle. It integrates all electric drive components in the vehicle body of a 1 Series Coupe while keeping the comfort of four full-fledged seats and a 200-litre luggage compartment. The electric motor develops a torque of $250 \mathrm{Nm}$ with a $170 \mathrm{hp}(125 \mathrm{~kW})$ engine. The range in real terms is up to $160 \mathrm{~km}$ (100 miles), depending on the driving style. As the battery is liquid cooled and able to be tempered, fluctuations due to high or low temperatures are expected to be reduced significantly. Charging times are comparable to the MINI E. Battery and drive train of the BMW ActiveE are pre-series versions of the BMW i3. In addition to standard laboratory and test track development procedures, these components are tested during the field trials in everyday customer environments. The customers did not participate as professional test drivers in this setting. When the vehicle is handed over to them, all safety and standard tests are fulfilled. By giving the car additionally to pilot customers it will be possible to make sure that scenarios and usage circumstances currently not known to test engineers will also be covered.

A test fleet of over 1,000 BMW ActiveE vehicles was sent out to provide this vital knowledge. Field trials with user research are conducted in Germany, the United States and China starting with the first pilot project involving 15 private and 15 fleet customers in Berlin in December 2011. Additional vehicles are used in Germany as part of governmentally funded research programs. Approximately 190 BMW ActiveE vehicles contribute to projects in Germany. The majority of the BMW ActiveE fleet is on the road in the United States since January 2012. About 700 vehicles are part of a project that has been created to gather not only customer experience but also market experience. Sales and handling processes are evaluated, options for add on services assessed and requirements on service infrastructure for large EV fleets examined. In 
early 2013 user research projects in China begin, involving roughly 100 BMW ActiveE cars. Additional vehicles are in the hands of private and corporate customers in France, the United Kingdom, the Netherlands, Italy, Switzerland, South Korea and Japan.

As the user research focus is clearly directed to a usability / user experience design approach, the HMI components of the BMW ActiveE play a much more central role than in the MINI E. Figure 2 gives an impression of the interior design. The instrument cluster and the iDrive control system and display functions were adapted for the first time in a BMW vehicle for electric vehicle specifics. The instrument on the right side of the cluster shows the amount of energy regenerated while slowing down the vehicle or the energy being consumed from the battery while accelerating. It also informs the driver that the vehicle is ready to drive as there is no engine noise normally connected to a driving-ready electric engine. The battery charging level and onboard computer information such as the remaining range are also displayed.
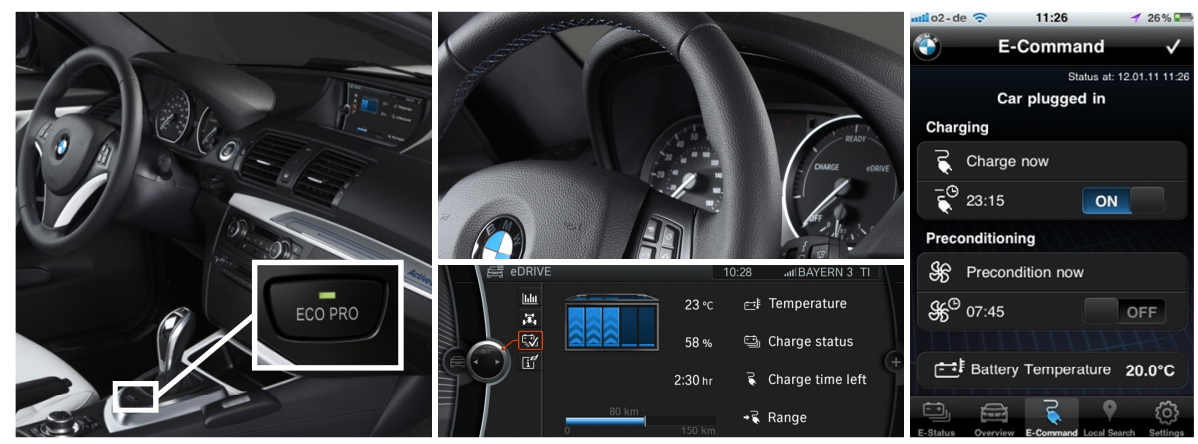

Fig. 2. BMW ActiveE interior (Fotocredit: BMW Group): Location of the ECO PRO button (left), instrument cluster and central display with vehicle energy information. Smartphone display of vehicle energy functions (right)

Further EV related functions are available via the Central Information Display. Schematic representations of the vehicle energy flows are designed to make electric mobility better perceptible and comprehensible. Specific sections inform about energy consumption of in-vehicle systems, range details and charging details like percentage available and time required for a full charge. Special charging functions are also implemented. The charge control function allows to start and finish the charging process and to program the charge timer. Preconditioning is a new function first introduced in the BMW ActiveE. It offers the possibility to cool or heat both the batteries and the vehicle interior. By bringing these components to the ideal operating temperature the highest performance of the energy storage is ensured and energy consumption during the trip is drastically reduced as the interior is already adjusted to a pleasant temperature without consuming energy from the battery. The ECO PRO mode is a new driving mode that allows to increase range by activating a switch in the center console. Efficient driving is then optimized by an adapted accelerator pedal characteristic delivering less power than in normal driving mode at identical pedal travels. Air conditioning, heating and ventilation systems are also reduced in energy consumption. In 
ECO PRO mode, the driver is additionally provided with tips on how to reduce energy consumption for best possible efficient driving.

In addition to onboard systems, several functions are available via BMW ConnectedDrive using direct internet connection, e.g. a charging station finder. With the "My BMW Remote" app, users are enabled to access functions remotely via smartphone. Besides standard functions like locating or locking the car, new remote functions for battery charge control and vehicle preconditioning as described above have been integrated.

Like in the MINI E, regenerative braking is initiated by taking the foot off the accelerator pedal. The strength of regenerative braking, $-1.8 \mathrm{~m} / \mathrm{s}^{2}$, was somewhat reduced compared to the MINI E. Using a distinctive intermediate position of the accelerator pedal, the driver is able to set the BMW ActiveE in a "glide" mode. The vehicle then acts like when in neutral / no gear mode and coasts freely with its gained momentum.

In the following, results from the BMW ActiveE field trials in Germany and the United States are described. Due to the ongoing development process of the series vehicle and due to the page restriction here, only a small subset can be presented.

\subsection{BMW ActiveE Field Trial Germany}

From December 2011 to March 2012 the first BMW ActiveE user study involving 15 private customers was undertaken. On average the customers' age was 46 (14 male, 1 female), ten of these customers were former MINI E drivers.

The study was planned and carried out in cooperation with the Institute of Cognitive and Engineering Psychology at the Chemnitz University of Technology and the market research agency Spiegel Institut Mannheim. It was designed to maximally share the customers' user experience with a three-phased research procedure. Customers were first contacted - besides initial interviews during application - after roughly 4 weeks in an open telephone interview stating their impressions in general as well as likes and dislikes. The drivers' experiences were directly fed into the planning of focus group sessions which were held after 8 weeks. Each customer participated in one of two focus groups mainly concentrating in one group on EV winter usage and preconditioning or in the other group on general system usability (esp. display and interaction concept) and efficient EV driving (including assistance functions). Besides this qualitative input, users gave additional feedback by taking part in an online survey at the end of the trial. The online survey incorporated questions from relevant development and strategy departments as well as those aspects that customers brought up during the focus groups which needed further clarification.

One of the most intriguing findings was that the ECO PRO driving mode was accepted very differently. Usage varied from 5\%-95\% share on total distance driven with the vehicle. Customers stated in the focus groups that some of them used it rather permanently while others only wanted a kind of take-me-home "emergency" mode in case of low battery capacity or large distances. Especially in the later case the drivers expect a more drastic effect on driving dynamics and comfort functions. Because of the large variability in usage patterns it was decided to seek support for this finding with the larger sample of US customers. 
The BMW ActiveE Berlin field trial took place completely during winter. Especially those customers with MINI E experience rated it very positively that even despite freezing temperatures electric driving was still possible. Although it took some time to get accustomed to using the preconditioning feature, it was welcomed after experiencing that it leads to range increase. However, several customers criticized that auxiliary systems like heating still reduced range considerably.

The option to monitor and control the charging process remotely was regarded to be an essential function for premium EV user experience. This holds especially for the preconditioning feature. Customers clearly showed a preference to use their smartphone to set the relevant parameters compared to carrying out the input operations in the vehicle.

When asking the ten former MINI E customers for a direct comparison, the BMW ActiveE was uniformly rated to be the more mature car which has a much higher potential to be used as the only car in the household. Several comparison dimensions are depicted in Figure 3. Although technologically less advanced it is interesting to see that the MINI E still was regarded as a potential candidate for the next vehicle purchase by three customers if the car was available on the market.

\begin{tabular}{l|r|l|l} 
& MINIE better & BMW ActiveE better & no difference \\
\hline Reliability in general & 2 & 5 & 3 \\
\hline Handling of charging hardware & 0 & 6 & 4 \\
\hline Candidate for next vehicle purchase & 3 & 5 & 2 \\
\hline Suitability as only car in household & 0 & 8 & 2 \\
\hline Suitability for winter use & 0 & 7 & 3 \\
\hline Regenerative deceleration & 5 & 3 & 2
\end{tabular}

Fig. 3. Direct comparison between the MINI E and the BMW ActiveE by 10 customers of the Berlin field trial with at least three months of usage experience with both vehicles

Regenerative braking was again a feature very much liked by the BMW ActiveE customers. As the MINI E field trials already revealed that individual preferences exist in the preferred level of the magnitude of deceleration when stepping off the accelerator pedal it is not surprising to see that several customers liked the MINI E deceleration characteristic better. Very important for future developments was that customers were able to use both, coasting and regenerative braking - depending on different use cases in deceleration and driving speed - as means of efficient driving.

\subsection{BMW ActiveE Field Trial USA}

The online survey from the Berlin field trial was extended with questions that either stemmed from development, sales or strategy departments or that were initiated by feedback in social media. Since the survey was still online during the creation of this 
document, preliminary findings based on $\mathrm{N}=79$ customers are presented that shed more light on the results presented above.

The results on the strength of regenerative braking are put on a broader basis. Did the comparison to the MINI E mean that regenerative deceleration definitely needs to be stronger? The majority of customers in the US rate the design of the BMW ActiveE's regenerative braking quite positively: While the majority was satisfied or even very satisfied (27\% and $67 \%$, respectively), only $5 \%$ were not satisfied (with $1 \%$ having no opinion).

Usage patterns of the ECO PRO mode are verified with a larger sample. As shown in Figure 4 the results of the focus group discussion are replicated. Again, a significant group of customers uses the ECO PRO mode as default while driving. On the other end, a large group of customers never uses the ECO PRO mode. Qualitative comments in the survey again point out, that a distinction between a "standard" energy saving mode and an "emergency use only" energy saving mode would make sense.

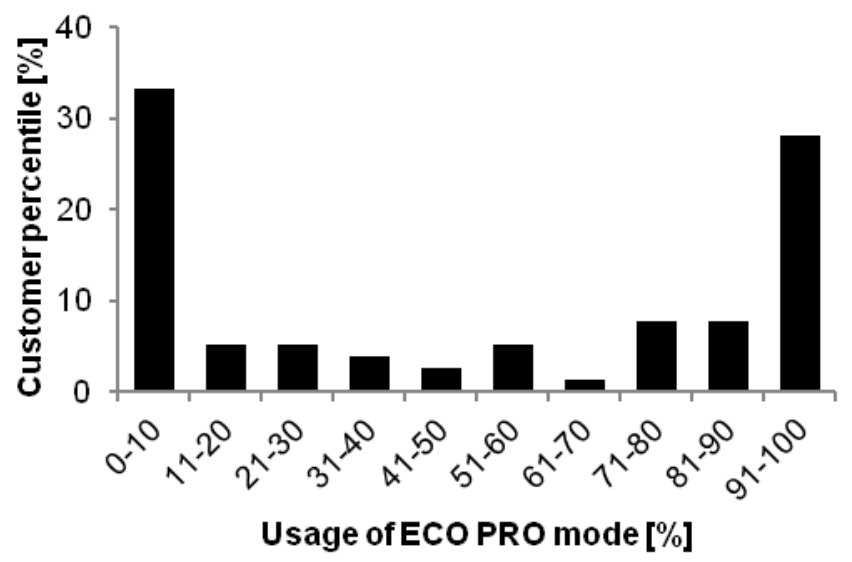

Fig. 4. Customers using ECO PRO mode as percentage of daily driving

Preconditioning was regarded to be a vital feature for future EVs. $82 \%$ of the drivers used this feature. The smartphone app did not work in all cases during the field trial which lead to customer complaints, providing further evidence for the importance of this option. The vast majority $(84 \%)$ required the availability via smartphone for the series vehicle.

\section{Conclusions and Future Developments}

The field trials provided ample evidence that EVs are suitable for everyday use. Based on customer feedback important functions were introduced or refined for the BMW i3. For instance, one-pedal driving with regenerative braking was identified as a key characteristic for positive user experience in e-mobility. The BMW i3 will feature different modes for energy saving, ECO PRO and the additional ECO PRO + mode which further reduces energy consumption to add crucial miles to the driving range if necessary in unforeseen circumstances. 
The field trials also demonstrated the necessity for functions that allow to better exploit the range available. The series vehicles will therefore come with advanced navigation functions that assist the driver by taking energy consumption based on driving characteristics into account as well as integrating geographical characteristics of route alternatives. Additional intermodal route planning options and so-called "last mile navigation" that continues to navigate the driver after already having parked the vehicle by sending instructions to the smartphone will make it easier to reach the final destination while charging the vehicle in a suitable location. Finally, in order to close any remaining mobility gap, BMW i will continue to develop fast charging technologies and mobility services targeting those use cases.

Acknowledgments. The authors would like to acknowledge the project $\mathrm{i}$ team at BMW for making the studies possible, especially Peter Krams with the project team involved in the Berlin field trial as well as Peter Dempster and Andreas Klein for their most valuable input in the US online survey. Thanks also to Juliane Schäfer for introducing social media analysis back in 2009 and to Andreas Keinath for contributing his research expertise during all trials. Most of all, in behalf of everybody involved in BMW's e-mobility projects, we would like to thank our pilot customers in the MINI E and BMW ActiveE field trials for their enduring support and inspiring feedback.

\section{References}

1. Klein, A., Spiegel, G.: Social Media in the Product Development Process of the Automotive Industry: A New Approach. In: HCI International 2013 Conference Proceedings. Springer, Heidelberg (accepted for publication, 2013)

2. Krems, J.F., Franke, T., Neumann, I., Cocron, P.: Research Methods to Assess the Acceptance of EVs - Experiences from an EV User Study. In: Gessner, T. (ed.) Smart Systems Integration. Proceedings of the 4th European Conference \& Exhibition on Integration Issues of Minituarized Systems. VDI Verlag, Como (2010)

3. Cocron, P., Bühler, F., Neumann, I., Franke, T., Krems, J.F., Schwalm, M., Keinath, A.: Methods of Evaluating Electric Vehicles from a User's Perspective - the MINI E Field Trial in Berlin. IET Intelligent Transport Systems 5, 127-133 (2011)

4. Vilimek, R., Keinath, A., Schwalm, M.: The MINI E Field Study - Similarities and Differences in International Everyday EV Driving. In: Stanton, N.A. (ed.) Advances in Human Aspects of Road and Rail Transport, pp. 363-372. CRC Press, Boca Raton (2012) 